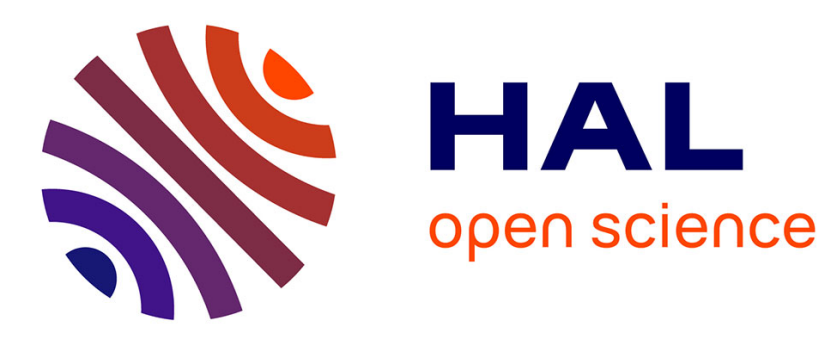

\title{
Marxisme et théorie néoclassique. La reconstruction incertaine de John Roemer \\ Fabien Tarrit
}

\section{To cite this version:}

Fabien Tarrit. Marxisme et théorie néoclassique. La reconstruction incertaine de John Roemer. Cahiers d'Economie Politique = Papers in political economy, 2020, 2020/2 (78), pp.27-53. 10.3917/cep1.078.0027 . hal-03090142

\section{HAL Id: hal-03090142 https://hal.science/hal-03090142}

Submitted on 29 Dec 2020

HAL is a multi-disciplinary open access archive for the deposit and dissemination of scientific research documents, whether they are published or not. The documents may come from teaching and research institutions in France or abroad, or from public or private research centers.
L'archive ouverte pluridisciplinaire HAL, est destinée au dépôt et à la diffusion de documents scientifiques de niveau recherche, publiés ou non, émanant des établissements d'enseignement et de recherche français ou étrangers, des laboratoires publics ou privés. 


\title{
Marxisme et théorie néoclassique. La reconstruction incertaine de John Roemer
}

\author{
Fabien Tarrit
}

\author{
REGARDS (URCA)
}

Résumé: Peut-on reconstruire sans la trahir la pensée économique de Marx à partir de la théorie néoclassique ? C'est la question à laquelle a tenté de répondre John Roemer. Il fut l'un des principaux contributeurs du marxisme analytique - plus précisément du marxisme de choix rationnel -, principalement au cours des années 1980. L'objectif fondateur de ce courant a été de parvenir à des conclusions proches de celles de Marx en utilisant des outils méthodologiques différents. Le programme de recherche de Roemer se fonde sur la théorie néoclassique. Nous discutons, d'un point devue critique, la cohérence et la pertinence de cette approche. Nous nous demandons si ce qui est remis en cause est la théorie de Marx ou la possibilité de l'associer aux outils de la théorie néoclassique.

Mots-clefs: Marx, valeur-travail, profit, exploitation, matérialisme historique

Abstract: CanMarx's economic thought be reconstructed from neoclassical economics? This is the question John Roemer tried to answer. He was a major actor of Analytical Marxism - especially of Rational Choice Marxism -, mainly during the 1980s. The founding objective of this school of thought has been to reach conclusions that are close to Marx's in using different methodological tools. Roemer's research program is based on the neoclassical theory. We shall discuss the consistency and the relevance of this approach through a critical examination. We wonder either what is questioned is Marx's theory or the possibility to associate it to the tools of the neoclassical theory.

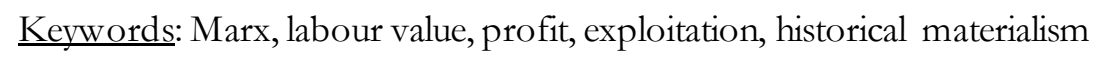

IEL: B24, B41, D50, P10

\footnotetext{
- Nous adressons nos plus vifs remerciements aux rapporteurs anonymes dont les commentaires ont contribué à une amélioration significative de la qualité de l'article.
} 
Aux côtés notamment de Jerry Cohen et de Jon Elster, John Roemer ${ }^{1}$ fut l'un des principaux contributeurs des travaux du Groupe de Septembre, c'est-à-dire du marxisme analytique ${ }^{2}$. Il nous semble opportun de distinguer, parmi ses travaux consacrés à l'œuvre de Marx, trois ouvrages majeurs : sur la théorie économique (1981), sur l'exploitation et le matérialisme historique (1982a) et sur la philosophie politique normative $(1988)^{3}$. Roemer estime que, dans la mesure où ils sont vieux d'un siècle, les outils de Marx ne sont pas compatibles avec la science sociale contemporaine. Dans la mesure où toute science qui hésite à oublier ses fondateurs est destinée à dégénérer, où la science physique ne se réfère plus à Galilée, où la microéconomie contemporaine n'est pas smithienne, le marxisme devrait écarter, ou pour le moins dépasser, sa référence à Marx. De la sorte, il affiche sa volonté de rompre avec la spécificité méthodologique du marxisme ${ }^{4}$, et de reformuler les débats en adoptant les normes dominantes du langage économique contemporain, à savoir l'approche néoclassique. "Ne connaissant aucune autre méthode, jutilise la méthode de l'équilibre» (Roemer 1981, p. 11), si bien que « [1] marxisme révisé que je présente ici est articulé par les perspectives que peuvent ouvrir les outils économiques contemporains, c'est-à-dire la théorie économique néoclassique $\|^{5}$ (Roemer 1988, p. vii). L'enjeu est pour lui de parvenir, en particulier sur la question de l'exploitation, à des conclusions théoriques similaires à celles de Marx tout en utilisant des outils différents. Il admet ne pas tenir un discours marxien, pour trois raisons (1982a). D'abord, en partant des postulats de la théorie économique néoclassique, il situe l'histoire hors de son champ d'analyse. Ensuite, les concepts qu'il utilise ne pas sont explicitement de Marx, mais il les présente comme une généralisation des concepts marxiens. Enfin, il ne fait aucune référence aux textes de Marx pour soutenir ses arguments. Il semble avoir pour objectif d'ouvrir les portes de l'université (américaine) au marxisme en adoptant le cadre du choix rationnel ${ }^{6}$, et c'est par les sujets qu'il aborde (exploitation, socialisme, communisme, lutte de classes...) qu'il se différencie de l'approche néoclassique. Se référant à Paul Lafargue ${ }^{7}$, et surtout s'appuyant sur les travaux de Michio Morishima ${ }^{8}$, John Roemer prétend conférer au marxisme le statut de science à l'aide des mathématiques. En proposant une formalisation mathématique des idées de Marx, Roemer se situe dans la continuité de la tradition économique (marxiste) japonaise - elle est relativement ancienne (années 1920) -, avec notamment les travaux de Kei Shibata $(1933,1934)$, dont les contributions portaient notamment sur les théories de la valeur et du profit. Il existe également une école japonaise plus récente portant le nom de marxisme analytique, elle-même articulée autour de la formalisation mathématique des œuvres de Marx?. Nous nous en tenons dans cet article aux contributions de Roemer à la fois pour saisir la mesure dans laquelle elles sont centrales dans ce débat et pour dégager combien elles interrogent explicitement la méthode marxienne avec le marxisme analytique. Elles permettent ainsi d'ouvrir de nombreuses pistes de réflexion ${ }^{10}$. Roemer définit le marxisme comme un modèle hypothético-déductif, c'est-à-dire un ensemble de postulats et de théorèmes soumis à examen rigoureux, à des tests de réfutation. C'est ainsi que de nombreuses positions marxistes sont révisées, notamment la théorie du profit. Il cherche à établir que des fondements microéconomiques sont compatibles avec le marxisme, avec comme unité la classe plutôt que l'individu, les individus agissant comme les agents d'une classe. D'une manière générale ses travaux, assez formalisés ${ }^{11}$, s'inscrivent dans un contexte intellectuel et institutionnel - l'université américaine, et plus généralement le monde académique anglophone - dans lequel la pensée marxiste n'a jamais eu d'influence significative ${ }^{12}$. Ainsi l'« approche [de Roemer] consiste à rejeter la théorie de la valeur travail comme théorie

\footnotetext{
${ }^{1}$ John Roemer, né en 1945, est américain. Diplômé de Harvard en mathématiques, il a obtenu un doctorat en économie à l'université de Berkeley en 1974. Il a par la suite enseigné l'économie à l'université de Davis et, depuis 2000, il est Professeur d'écono mie et de sciences politiques à l'université de Yale. Depuis 2006, il est membre, tout comme Elster, de l'Académie Américaine des Arts et des Sciences.

${ }^{2}$ La contribution de Roemer correspond à un marxisme de choix rationnel, qui peut être conçu comme un sous-ensemble du marxisme analytique. Voir Tarrit, 2014.

${ }^{3}$ Cet article présente une synthèse de ces ouvrages et les situe dans leur contexte intellectuel.

${ }^{4}$ Le renversement matérialiste de la dialectique hégélienne, et en particulier son application à l'histoire, fondent la spécificité méthodologique de la pensée de Marx.

5 Toutes les citations de textes en anglais sont traduites par nos soins.

6 «Pourquoi donner au diable les meilleures armes ?» (Roemer 1986, p. 192-193)

7 «Une science n'est développée que lorsqu'elle a atteint la capacité d’utiliser les mathématiques » (Lafargue 1891, p. 13).

8 Il faut « reconnaître en Marx un économiste mathématicien » (Morishima 1974, p. 694).

${ }_{9}$ Voir Takamasu, Matsui, 1999

10 À ce titre nous pouvons nous référer aux prolongements auxquels il a donné lieu, dans le même esprit (Yoshihara, Veneziani 2018), ou dans une logique de dépassement (Wright 1985, Vrousalis 2013).

11 Ils sont présentés ici de manière littéraire.

12 «S'il existe un seul professeur aux États-Unis qui enseigne l'économie politique et se dise socialiste, ce professeur est une aiguille que je n'ai pu trouver dans notre botte de foin universitaire » (Sinclair, 1923, p. 436) et « on constate [...] un fait curieux, l'absence d'un marxisme anglais » (Elster 1981, p. 745).
} 
de l'échange, et à reconstruire la théorie de l'exploitation sur une base différente » (Roemer 1981, p. 52). Nous la présentons d'un point de vue critique en nous demandant si une telle reformulation de la théorie économique marxiste doit être conçue comme une remise en cause de cette théorie ou comme la possibilité de l'associer aux outils de la théorie néoclassique, ce qui dans les deux cas interroge dans la spécificité de la méthode de Marx. Ainsi, à partir d'une déconstruction de la théorie marxienne de la valeur et d'une réfutation de la loi de la baisse tendancielle du taux de profit (1), Roemer propose une redéfinition de la théorie de l'exploitation, qu'il inscrit également dans le matérialisme historique (2).

\section{Une critique des théories marxiennes de la valeur et du profit}

En proposant l'attribution de fondements microéconomiques à la théorie de Marx ${ }^{13}$, les premiers travaux de John Roemer portent un regard critique sur les fondements économiques du marxisme. Une critique radicale de la théorie de la valeur travail le conduit à préconiser son abandon (1.1), et il en résulte une réfutation de la loi de la baisse tendancielle du taux de profit (1.2).

\subsection{Une déconstruction de la théorie de la valeur travail}

Pour Roemer, la théorie de la valeur travail pénalise la pensée économique marxiste, qui doit être reconstruite indépendamment de cette théorie. Les grands traits de son approche sont les suivants : d'une part il nie la spécificité de la force de travail comme unique marchandise créatrice d'un surplus de valeur, ce qui en l'occurrence correspond à une lecture microéconomique de la valeur (1.1.1); d'autre part il inverse le sens de la causalité sur la question de la transformation (1.1.2).

\subsubsection{Une lecture microéconomique de la valeur}

Pour parvenir à son objectif - reconstruire la théorie marxiste, ou au moins certains de ses concepts, avec les outils la théorie néoclassique -, Roemer fait l'économie de la théorie de la valeur travail14. Alors que pour Marx, la réelle source de valeur est le travail appliqué à la nature - en ce que toutes les marchandises ont en commun une propriété, celle d'être produites par du travail -, le travail n'est pour Roemer qu'une source de richesse parmi d'autres : toutes les marchandises ont en commun d'être désirées, et leur détention fournit un bien-être. Il est donc possible, selon lui, de formuler une théorie de la valeur fondée sur l'utilité. De la sorte, il généralise le «théorème marxien fondamental » proposé par Nobuo Okishio ${ }^{15}$ (1961) puis énoncé par Michio Morishima (1973) ${ }^{16}$. Il affirme que le travail est une marchandise semblable aux autres, qu'il n'a pas l'exclusivité de pouvoir servir de numéraire et donc d'être exploité17. «La force de travail [...] n'est pas le seul facteur productif doté d'une propriété d'exploitabilité » (Roemer 1988, p. 53), « [c] et énoncé est [...] vrai à propos du maïs, de l'acier ou de l'énergie »(Roemer 1982a, p. 185). Il formule un théorème généralisé d'exploitation de marchandises qui suggère qu'il existe un profit si et seulement si ces marchandises possèdent une propriété d'exploitabilité18 lorsqu'elles sont utilisées comme numéraire pour le calcul de la valeur incarnée ${ }^{19}$. Il deviendrait alors possible de distinguer par exemple le taux d'exploitabilité de la force de travail (surtravail par unité de force de travail / quantité de travail nécessaire à la reproduction de cette unité) et le taux d'exploitabilité du maïs ${ }^{20}$ (surplus de maïs par unité de maïs / montant de maïs nécessaire à la reproduction de cette unité), sachant que « le taux d'exploitabilité peut être conçu comme une mesure de l'efficacité productive d'un facteur » (Roemer 1988, p. 53).

\footnotetext{
13 «Ce type de raisonnement économique, la déduction d'effets économiques globaux à partir du comportement d'unités économiques individuelles, fut employé par des économistes de toute tendance idéologique au dix-neuvième siècle. Il s'agit en fait d'un des signes pour lesquels le marxisme est le socialisme scientifique» (Roemer 1981, p. 112).

${ }^{14}$ De même que Morishima propose « une économie marxienne sans théorie de la valeur travail» (1973, p. 181), Roemer souhaite « reconstruire les concepts marxistes d'une manière qui les débarrasse de [...] la théorie de la valeur travail »(1988, p. 173).

${ }^{15}$ Notons qu'Okishio s'est largement appuyé sur les résultats de Kei Shibata $(1933,1934)$.

16 "L'exploitation des travailleurs par les capitalistes est une condition nécessaire et suffisante à l'existence d'un ensemble de prix et de salaires garantissant des profits positifs ou, en d'autres termes, à la possibilité de maintenir l'économie capitaliste » (Morishima 1973, p. 53).

17 Roemer reprend la critique néo-ricardienne du Capital. Alors que pour Marx, l'exploitation correspond à une extraction de surtravail dans le processus de production, il s'agit pour les néo-ricardiens d'un mode de distribution du produit social (Steedman 1977).

${ }^{18}$ Une marchandise possède «la "propriété d'exploitabilité” [si] une unité de [cette marchandise] peut être socialement reproduite avec moins d'une unité» (Roemer, 1988 p. 53).

${ }^{19}$ Roemer ne distingue pas la mesure de la valeur et son origine.

${ }^{20}$ Il s'agit de l'exemple traditionnellement utilisé par Roemer. Voir Hervier 1995.
} 
Roemer envisage la théorie de la valeur travail sous l'angle de la microéconomie, c'est-à-dire en analysant le travail à l'échelle de l'individu. En ce sens, il s'éloigne de la conception par Marx du travail socialement nécessaire comme moyenne du travail social. Reprenant les positions de Morishima (1973) et de Steedman (1977), il estime que la plus-value sociale n'est pas une agrégation des plus-values individuelles. Ceci le conduit à estimer que les différences qualitatives entre plusieurs types de travaux conduisent à leur incommensurabilité, et qu'il est impossible d'obtenir une théorie de la valeur travail du fait de cette hétérogénéité. Ainsi la théorie de l'exploitation doit être construite indépendamment de la valeur travail, conçue comme un concept microéconomique de valeurs individuelles. En outre, non seulement Roemer attribue à tort la théorie malthusienne du salaire de subsistance à Marx ${ }^{21}$ - c'est ce qu'il suggère en affirmant qu'« il devient nécessaire de définir un concept (marxien) d'exploitation qui soit indépendant de toute notion de subsistance » (1982a, p. 110) - mais il estime également qu'elle est indispensable à la théorie de la valeur travail - une position partagée par Morishima ${ }^{22}$ et par les néo-ricardiens -, en même temps qu'elle est tautologique En prêtant ainsi à Marx une théorie du salaire de subsistance, il s'expose précisément au même reproche que celui adressé par Marx à Lassalle dans sa critique du programme de Gotha à propos de sa « loi d'airain sur les salaires ", qu'il juge malthusienne. Pour Marx, la question n'est pas tant le niveau du salaire que le niveau de surtravail et donc de plus-value : «l'ouvrier salarié n'est autorisé à travailler pour assurer sa propre existence $[. .$.$] qu'autant qu'il travaille gratuitement un certain temps pour les capitalistes » (Marx et$ Engels 1875, p. 39). Il sépare le salaire en une composante biologique, correspondant au minimum nécessaire pour la reproduction et l'entretien de la force de travail, et une composante socio-historique, correspondant aux acquis historiques de la classe ouvrière. Roemer juge quant à lui qu'« [i]l n'est pas nécessaire de discuter si la "subsistance" est un concept biologique ou historique » (1988, p. 153). En conséquence il renonce à cette loi, qu'il qualifie de «théorie spéciale de la subsistance », et il la remplace par une "théorie générale de la lutte de classes", seule à même selon lui de reformuler une théorie de l'exploitation qui ne soit pas fondée sur la théorie de la valeur travail. Notons toutefois que la subjectivité des travailleurs de Roemer est relativement pauvre et rien ne lui permet d'envisager qu'ils développent entre eux, autrement que sur un mode déterministe strictement lié à leur dotation, une solidarité systémique, ou qu'apparaisse un antagonisme structurel avec les capitalistes. Pour Roemer, Marx aurait choisi le travail comme numéraire, d'une part pour des raisons normatives et non scientifiques (c'est une marchandise inaliénable qui prend en compte les rapports entre les hommes car tous sont dotés de force de travail), d'autre part pour correspondre au matérialisme historique ('’histoire est l'histoire de la lutte de classes).

Cette approche comporte un certain nombre d'implications sur la question de la transformation.

\subsubsection{Sur la question de la transformation}

La question de la transformation de la valeur en prix a fait l'objet d'une multitude de débats entre économistes ${ }^{23}$. Elle nous parait périphérique quant à la pensée économique de Marx ; plus précisément nous partageons la position qu'elle est un «non-problème» (Kliman et McGlone 1988, p. 56), au sens où dans une large mesure «le débat [...] sous-évalue ou même ignore la question de la méthode [et relève d'une] compréhension non dialectique » (Ibid., p. 57). En toute logique, compte tenu de son rejet de la méthode dialectique, elle est d'une grande importance pour Roemer. Il en fait même «le sujet de l'ensemble des livres du Capital» (1981, p. 159). Pour lui elle dépasse largement la relation entre valeur et prix et peut selon lui "s'exprimer comme autant de moules qu'il existe de disciplines en sciences sociales » (Ibid.), d'où le découpage suivant : transformation sociologique (fétichisme de la marchandise), transformation historique (évolution des modes de production), transformation philosophique (relation causale entre le monde des prix et le monde social) et transformation économique (entre valeurs et prix). Or il considère que ce qui a été écrit portait principalement sur la dimension économique, à savoir la relation d'une part entre valeur et prix et d'autre part entre exploitation et plus-value et entre profits et réalisation des profits. Dans la continuité logique de son rejet de la théorie de la valeur travail, Roemer considère également que le problème de la relation entre valeur et prix est un «non-problème» (1981, p. 160), mais pour une raison radicalement opposée à celle de Kliman et McGlone. Pour Roemer, elle est « un projet erroné, dont l'origine repose sur un effort de penser que les valeurs travail jouent un rôle important dans la régulation des échanges » (Ibid.).

\footnotetext{
${ }^{21}$ Elle fut reprise par Ferdinand Lassalle : «Le salaire garde toujours comme fondement nécessaire la subsistance, ce qui est normalement requis pour l'existence et la reproduction» (1863 p. 15-16).

22 «À supposer chaque travailleur payé au niveau de subsistance (hypothèse de base chez Marx) " (Morishima, 1974 p. 698).

${ }^{23}$ Pour une synthèse récente de ces débats, voir Saad-Filho 2012.
} 
Les prix sont déterminés avant la définition de la valeur, dans la mesure où « la valeur travail dépend du marché » (Ibid., p. 203). Nous avons vu que c'est en s'appuyant sur les travaux de la tradition marxiste japonaise que Roemer, en interprétant la théorie de la valeur travail d'un point de vue microéconomique, conclut à son impossibilité, ce qui correspond également à une indépendance entre valeurs et prix. Une telle critique néo-ricardienne de la valeur, ainsi que son affirmation de l'indépendance entre prix et valeurs, vont naturellement impliquer une nouvelle lecture de la théorie marxienne du profit.

\subsection{Une réfutation de la loi de la baisse tendancielle du taux de profit}

Dans le prolongement logique de la critique qu'il adresse à la théorie marxienne de la valeur travail, Roemer cherche à réfuter, sur des fondements méthodologiques néoclassiques, la loi de la baisse tendancielle du taux de profit, qui selon lui est une faiblesse de la théorie économique marxienne - son « calice » (Roemer 1981, p. 88), c'est-à-dire une limite majeure de la théorie, qu'il compare à l'incapacité de la théorie néoclassique à " fournir une explication satisfaisante du chômage dans une économie de marché » (Ibid., p. 89). Se fondant sur des postulats microéconomiques, il défend la possibilité d'une hausse continue du taux de profit, si bien que la loi serait réfutée par ses contre-tendances. À l'encontre de la tradition marxiste, Roemer estime nécessaire d'étudier les fondements microéconomiques du comportement, sans pour autant rejeter le marxisme. «Parce que pour nombre de marxistes, la technique consistant à explorer les "fondements micro" du comportement économique peuvent sembler être une méthodologie néoclassique (et donc interdite), il faut insister sur le fait que ce n'est pas le cas. C'est en effet une des propriétés de l'analyse marxiste qui la rendent scientifique et non utopique » (1981, p. 114).

C'est par exemple le cas de la théorie de la baisse du taux de profit, telle qu'exposée par Marx dans le Livre III du Capital; elle est conçue comme un phénomène macroéconomique résultant du comportement concurrentiel d'unités économiques individuelles. Pour maximiser son profit individuel, chaque capitaliste substitue du capital au travail, ce qui conduit à augmenter la composition organique du capital, et donc à la baisse du taux de profit général. Roemer considère cette théorie comme un déterminisme technologique, un matérialisme vulgaire. Le dogmatisme qu'il attribue à la hausse de la composition organique du capital et à la baisse du taux de profit aurait empêché le développement créatif du marxisme, qui doit donc s'en débarrasser.

Par exemple, pour Roemer, une innovation technique introduite par les capitalistes en raison d'une baisse des coûts conduira - avec prix concurrentiels et salaire réel fixe -à une hausse du taux de profit. Il distingue des considérations techniques et des considérations sociales, et il estime que le progrès technique, en permettant d'augmenter le taux d'exploitation, permet de surcompenser la hausse de la composition organique du capital, et donc d'augmenter le taux de profit, ce qui serait toujours le cas, avec l'hypothèse d'un salaire réel stable. Il reprend les résultats d'Okishio (1961) selon qui le taux de profit augmente pour certaines catégories de changement technique. En cas d'innovation technique, et donc de diminution de la valeur des imputs utilisés ou de baisse des coûts, le taux de profit augmente. Par conséquent, seuls des éléments sociaux, jugés extra-économiques, peuvent conduire à la baisse du taux de profit. «Si le taux de profit diminue dans le capitalisme concurrentiel, ce doitêtre dû à une augmentation du salaire réel » (Roemer 1981, p. 108). Pour Roemer, la question est donc sociale et non structurelle. Une éventuelle diminution du taux de profit serait causée, non pas par une hausse de la composition organique du capital, mais par une diminution du taux d'exploitation. Chez Marx, il s'agit de contre-tendances à la baisse du taux de profit ${ }^{24}$. Roemer refuse une telle loi, en estimant que les profits relèvent d'un surplus social authentique reposant sur des rapports de propriété eux-mêmes garantis par des institutions, et pas d'une loi économique.

Roemer aborde également la question de la péréquation des taux de profit. Nous pouvons résumer la position traditionnelle en affirmant qu'à travers la concurrence, un transfert de capitaux s'opère vers les secteurs à plus fort taux de profit, ce qui conduit à l'égalisation des taux de profit. Pour sa part, Roemer estime que les taux de profit ne s'égalisent pas, pour deux raisons : d'une part ils sont différents si la productivité pour un même bien diffère entre capitalistes individuels, d'autre part l'existence de rendements décroissants peut conduire à des taux de profit différentiels (avec un profit total en hausse mais un taux de profit en baisse). Il s'appuie là encore sur les travaux de Morishima, mais également sur ceux de Steedman

\footnotetext{
${ }^{24}$ Les moyens de contrecarrer la baisse du taux de profit énoncés par Marx (1894, tome I, p. 245-253) sont : une augmentation du degré d'exploitation du travail, une réduction du salaire en dessous de sa valeur, une baisse du prix des éléments du capital constant, la surpopulation relative, le commerce extérieur, l'augmentation du capital par actions.
} 
(1977) et il affirme que la plus-value sociale n'est pas une agrégation des plus-values individuelles. Il existe des compositions organiques du capital différentes entre les secteurs, le taux de profit n'est pas uniforme et la théorie de l'échange du tome I du Capital ne fonctionne que pour un cas particulier, celui du travail homogène ${ }^{25}$, ce qui est une hypothèse de base des modèles néoclassiques.

Malgré l'affirmation de Marx selon laquelle la loi de la baisse tendancielle du taux de profit est la « loi la plus importante de toute l'économie politique moderne » (Marx 1894, tome I, p. 275), il apparait que son rejet est le seul élément de la théorie économique sur lequel les marxistes analytiques sont parvenus à un accord général ${ }^{26}$. Cet élément de la théorie économique marxienne est, d’un avis largement partagé par les auteurs, le seul pouvant être analysé à l'aide des outils de la théorie du choix rationnel. L'échec qui lui est attribué est largement perçu comme celui de la théorie de Marx et plus globalement de la possibilité de toute tendance supra-individuelle. Il serait donc impossible d'inscrire la théorie de Marx dans la logique des résultats non intentionnels d'actions intentionnelles, propres à la théorie du choix rationnel. Pour Roemer, il faut abandonner les théories de la valeur et de la plus-value, et donc du taux de profit. En revanche, il semble plus indulgent avec la théorie de l'exploitation.

\section{Une transformation de la théorie de l'exploitation}

«Les théories de la valeur et de la plus-value doivent être abandonnées. Mais ce n’est pas le cas de la théorie marxiste de l'exploitation qui peut être construite indépendamment de la théorie de la valeur travail comme théorie quantitative de l'échange » (Roemer 1981, p. 149). Sa contribution a été jugée comme «la tentative la plus importante depuis Marx, par sa rigueur et son ampleur, visant à faire progresser la théorie de l'exploitation » (Fleurbaey 1990, p. 116). En s'appuyant sur la distinction positiviste entre analyse scientifique et préoccupations normatives, il fonde la théorie de l'exploitation sur l'équité, au sens où elle est centrale pour juger un système, du point de vue de la population : «Lorsque la population soutient le capitalisme en tant que système, je pense que ce n'est pas seulement parce qu'elle pense que le capitalisme distribue les biens mieux que d'autres systèmes mais aussi parce qu'elle pense que dans un système capitaliste les personnes méritent ce qu'elles reçoivent» $(1988$, p.3). En séparant une conception technique d'une conception éthique de l'exploitation, et après avoir supprimé les fondements scientifiques de la théorie (théorie de la valeur travail et loi de la baisse tendancielle du taux de profit), il se rapproche des théories radicales contemporaines en philosophie politique, de même que Cohen ${ }^{27}$. Pour Roemer, la position marxiste s'appuie sur des fondements éthiques et la théorie marxienne de l'exploitation est mieux conçue comme une théorie normative. Il estime que la définition en termes de retrait ${ }^{28}$ - théorie des jeux - est supérieure à la définition en termes de plus-value. Elle clarifie les "impératifs éthiques » de la théorie marxienne, elle permet de démontrer que les riches exploitent effectivement les pauvres. Roemer ne nie pas l'existence d'une lutte, mais il la considère comme un phénomène exogène. Il estime que l'exploitation ne repose pas sur le surtravail, mais sur les rapports de propriété. Ainsi, elle n'est pas une définition, une caractéristique propre au capitalisme, mais un théorème à prouver. Il inverse rapports de production (infrastructure) et rapports de propriété (superstructure) dans leur ordre logique, en remplaçant la primauté explicative des rapports de production capitalistes par la propriété différentielle des biens productifs. Sa reconstruction de la théorie de l'exploitation est indépendante de la théorie de la valeur travail (2.1), et c'est en s'appuyant sur les travaux de Cohen qu'il établit cette reconstruction en coordinationavec le matérialisme historique (2.2).

\subsection{Une exploitation sans travail}

Pour Roemer, il est empiriste de fonder l'exploitation sur la théorie de la valeur travail. L'échange de travail n'est pas au cœur de son analyse de l'exploitation, qui ne nécessite pas de rapport entre exploiteur et exploité. Roemer élabore une théorie générale de l'exploitation (1982a), dans laquelle la théorie de l'exploitation capitaliste est un cas particulier. En se fondant sur une distinction entre échange de travail coercitif (esclavagisme puis féodalisme) et échange de travail non coercitif (capitalisme puis socialisme), il étudie

\footnotetext{
25 « Il ne peut pas exister de généralisation correcte de l'exploitation marxienne en cas de travail hétérogène » (Roemer 1982a, p. 183).

${ }^{26}$ En effet, aucun marxiste analytique n'accepte cette loi, et seul Robert Brenner accepte la théorie de la valeur travail.

27 « Poser la question de l'exploitation revient à poser la question du statut moral de la propriété privée capitaliste » (Cohen 1983, p. 316).

${ }^{28}$ Un agent est exploité si sa situation est plus favorable lorsqu'il se retire du jeu, c'est-à-dire en conservant ses dotations.
} 
comment un processus similaire d'enrichissement est possible dans les deux cas. Sa théorie a notamment pour objectif d'expliquer ce qu'il considère comme un rapport d'exploitation dans les États dits socialistes, et qui lui apparait comme une anomalie dans la théorie marxiste - le socialisme est censé se caractériser par l'absence d'exploitation. En conséquence, il considère que, pour construire une théorie de l'exploitation solide, il faut relâcher l'hypothèse de propriété privée des moyens de production. Ainsi, les causes institutionnelles de l'exploitation marxienne sont, selon lui, l'existence de marchés concurrentiels, la propriété différentielle des moyens de production, les différences de préférences et de niveaux de technologie, plutôt que l'expropriation directe du travail. Il estime que la caractéristique fondamentale du capitalisme est la dotation différentielle des moyens de production. Fondée sur son rejet de la théorie de la valeur travail, sa théorie de l'exploitation repose sur des fondements microéconomiques (2.1.1) et s'appuie sur la théorie des jeux (2.1.2).

\subsubsection{Une exploitation microéconomique : correspondance et isomorphisme}

Sur les traces de Morishima, Roemer cherche donc à construire une théorie de l'exploitation indépendamment de la théorie de la valeur travail ${ }^{29}$ et l'envisage comme le résultat d'un processus d'optimisation. Dans le cadre initial tout commence avec une économie de subsistance précapitaliste sans marché du travail. Les agents qui y interviennent sont tous des producteurs-consommateurs qui ont les mêmes préférences, la même capacité de travail, et recourent à la même technologie de production. Ils diffèrent uniquement dans leurs dotations initiales en biens. Chacun utilise sa dotation en biens et son propre travail pour produire des biens qu'il pourra échanger sur le marché des biens afin d'obtenir son panier de subsistance (qui est également identique pour tous). Il est, en particulier, montré que pour des producteurs qui minimisent leur temps de travail, certains travailleront beaucoup plus que d'autres. Cette situation est observée quand les dotations initiales sont inégalitaires : ceux qui sont riches en dotations auront plus facilement accès à la production que ceux qui sont pauvres en dotations et, par conséquent, les pauvres en dotations devront travailler plus longtemps pour obtenir leur panier de subsistance. Ainsi, même en l'absence d'un marché du travail, l'exploitation au sens marxiste (pour laquelle certains agents s'approprient le surtravail des autres) est observée.

Le modèle est élargi en y introduisant un marché du travail. Il est alors montré que les producteurs qui ont de faibles dotations (ici la richesse) vendront leur force de travail et seront exploités tandis que ceux qui ont de fortes dotations achèteront de la force de travail et seront exploiteurs. Ce résultat est appelé le " principe de correspondance classe-exploitation ». "Ce théorème [...] modélise et vérifie formellement une intuition marxienne centrale - selon laquelle l'appartenance à une classe qui embauche le travail est associé à l'exploitation, et l'appartenance à une classe qui vend du travail est associée au fait d'être exploité » (Roemer 1982a, p. 15).

Plus précisément, il existe trois formes possibles d'activité sur le marché du travail : produire pour soi, embaucher de la force de travail pour produire, vendre sa propre force de travail contre un salaire. Chaque producteur optimise en combinant ces trois activités, ce qui permet à Roemer de dégager cinq classes sociales : capitaliste pur, petit capitaliste, petit-bourgeois, semi-prolétaire, et prolétaire.

\begin{tabular}{|l|c|c|c|}
\hline & $\begin{array}{c}\text { Produit pour } \\
\text { soi }\end{array}$ & $\begin{array}{c}\text { Embauche de la } \\
\text { force de travail }\end{array}$ & $\begin{array}{c}\text { Loue sa force de } \\
\text { travail }\end{array}$ \\
\hline Capitaliste pur & Non & Oui & Non \\
\hline Petit capitaliste & Oui & Oui & Non \\
\hline Petit-bourgeois & Oui & Non & Non \\
\hline Semi-prolétaire & Oui & Non & Oui \\
\hline
\end{tabular}

\footnotetext{
29 «La théorie de l'exploitation chez Marx ne peut survivre à la révolution Von Neumann que dans une économie à travail homogène » (Morishima 1973, p. 96). Comparer avec Roemer, note 25.
} 


\begin{tabular}{|l|l|l|l|}
\hline Prolétaire & Non & Non & Oui \\
\hline
\end{tabular}

D’après Roemer, 1982a

Un capitaliste pur est suffisamment bien doté en capital pour ne pas avoir à travailler, il embauche de la force de travail. Un petit capitaliste est bien doté en capital, mais insuffisamment pour vivre exclusivement du travail des autres; il embauche de la force de travail et travaille également sur son propre capital. Un petit-bourgeois est bien doté en capital, mais insuffisamment pour embaucher, il travaille uniquement sur son propre capital. Un semi-prolétaire est faiblement doté en capital il travaille sur son propre capital et vend sa force de travail. Un prolétaire n'est pas doté de capital, il vend sa force de travail. Ce modèle considère également les positions intermédiaires puisque trois classes apparaissent entre la bourgeoisie et le prolétariat. Il tend ainsi à montrer que les classes sociales sont liées à la présence d'un marché du travail. Mais cela n'est vrai qu'en apparence.

En effet Roemer reprend la même analyse en considérant non plus un marché du travail mais un marché du crédit - ou du capital - où les prêteurs et les emprunteurs remplacent les employeurs et les salariés. Il en ressort un résultat parfaitement équivalent au précédent: les personnes faiblement dotées en capital emprunteront du capital et seront exploitées (c'est-à-dire qu'ils effectueront un surplus de travail), tandis que ceux qui ont de fortes dotations en capital se contenteront de prêter (sans avoir à produire car ils peuvent vivre des intérêts qu'ils perçoivent) et seront des exploiteurs.

Cette construction lui permet d'aboutir au théorème de l'isomorphisme, selon lequel le marché du capital (« île 30 $^{30}$ du marché du crédit ») possède la même structure que le marché du travail (" île du marché du travail») et comprend également cinq classes sociales: prêteur pur, prêteur mixte, producteur indépendant, emprunteur mixte, emprunteur pur.

\begin{tabular}{|l|c|c|c|}
\hline & $\begin{array}{c}\text { Produit pour } \\
\text { soi }\end{array}$ & Prête & Emprunte \\
\hline Prêteur pur & Non & Oui & Non \\
\hline Prêteur mixte & Oui & Oui & Non \\
\hline $\begin{array}{l}\text { Producteur } \\
\text { indépendant }\end{array}$ & Oui & Non & Non \\
\hline Emprunteur mixte & Oui & Non & Oui \\
\hline Emprunteur pur & Non & Non & Oui \\
\hline
\end{tabular}

Un prêteur pur est suffisamment bien doté en capital pour pouvoir se contenter de prêter (et de vivre des intérêts) sans avoir à produire lui-même. Un prêteur mixte est suffisamment bien doté en capital pour pouvoir prêter, mais il doit également produire pour assurer sa subsistance. Un producteur indépendant est doté en capital mais il ne prête pas et produit lui-même. Un emprunteur mixte travaille en partie pour luimême mais, étant faiblement doté en capital, il est contraint d'emprunter. Un emprunteur pur n'a aucun capital, il ne peut qu'emprunter.

L'île du marché du travail a pour fonction la vente de la force de travail, lî̀le du marché du crédit a pour fonction la location des moyens de production. Elles sont identiques dans leur structure : chaque agent est exploité de la même manière que son homologue sur l'autre île. L'exploitation est la même dans les deux cas, d'où le théorème d'isomorphisme de Roemer qui établit une équivalence entre la vente de force de travail et l'emprunt de capital. L'exploitation peut avoir lieu via l'échange de marchandises et les classes peuvent exister avec un marché du crédit sans marché du travail. En d'autres termes, les classes sociales, et

\footnotetext{
30 John Roemer est également usager des «robinsonnades » déjà critiquées par Marx. "Le chasseur ou le pêcheur singulier et singularisé, par lequel commencent Smith et Ricardo, ressortit aux plates illusions des robinsonnades du $18^{\mathrm{e}}$ siècle, lesquelles n'expriment nullement, comme se l'imaginent certains historiens de la civilisation, une simple réaction contre des excès de raffinement et un retour à l'état de nature mal compris » (Marx 1858, tome I, p. 17).
} 
par là même l'exploitation, peuvent exister avec un marché du capital et sans marché du travail. Cela nous amène au principal message de Roemer qui tranche avec la vision classique de l'exploitation : "La caractéristique fondamentale del'exploitation capitaliste n'est pas ce qui se passe dans le processus de travail, mais la propriété différentielle des actifs productifs» (1982a, p. 94-95). Il suffit que la coercition ait lieu au niveau des rapports de propriété plutôt qu'au niveau des rapports de production, qui possèderaient une importance secondaire. Il en conclut que « [s] l l'exploitation du travailleur est un concept important, il l'est pour des raisons normatives - parce qu'elle constitue un indicateur d'une certaine fore d'injustice, et pas parce que l'exploitabilité de la force de travail est l'unique source de profit» (1988, p. 54), ce qui est compatible avec son rejet de la théorie de la valeur travail, et fait de l'exploitation un enjeu de philosophie politique. Pour Roemer, aucune raison objective ne pousse à privilégier le travail comme référence de valeur, les théories de la valeur ne peuvent pas prétendre à l'autonomie, et l'exploitation correspond à une inégalité de revenu, liée à une dotation injuste de biens productifs. Dans un cadre hypothétique où l'inégalité originelle n'est pas injuste, l'exploitation porteuse de cette inégalité ne serait pas injuste.

\subsubsection{Exploitation et théorie des jeux}

La notion d'exploitation est conçue en termes de théorie des jeux. Précisons qu'il s'agit d'une approche spécifique de la théorie des jeux, à savoir la théorie des jeux coopératifs, d'où un raisonnement en termes de coalition. Elle présuppose une allocation alternative utilisée comme critère d'évaluation de l'allocation existante. Une coalition est dite exploitée pour une allocation donnée si sa rémunération était meilleure dans l'allocation alternative que dans l'allocation présente. Il dégage trois conditions pour que l'exploitation d'une coalition $S$ par la coalition $S$ 'ait lieu dans une société $N$ (Roemer 1982a,p. 194-195) : il existe une alternative réalisable dans laquelle la situation de $S$ serait plus favorable; dans cette alternative, la situation de $S$ ' (complément de $S$ ) serait moins favorable; $S$ ' exerce une domination sur $S$ dans $N$. $S$ aurait ainsi intérêt à se retirer de $\mathrm{N}^{31}$. Ainsi, l'exploitation est perçue comme le résultat d'une stratégies d'acteurs cherchant à optimiser leur situation ${ }^{32}$. Ici, Roemer remplace le surtravail par les rapports de propriété et il affirme que l'exploitation peut logiquement exister sans échange de travail.

Une telle représentation de l'exploitation a donné lieu à d'abondants commentaires, principalement en langue anglaise ${ }^{33}$. Il lui est notamment attribué une lecture superficielle (Anderson, Thompson 1988), une incompréhension du caractère dialectique de la pensée de Marx (Smith 1989), il lui est reproché d'absorber la pensée marxiste dans un cadre antimarxiste (Lebowitz 1988; Hunt 1992), de ne pas tenir compte de la réalité sociale (Kieve 1986; Burris 1988), de ne pas saisir l'instabilité dynamique du capitalisme (Callinicos $2002)^{34}$... Notons que son approche ne distingue pas le capitalisme des rapports précapitalistes. À l'inverse, pour Marx, il s'agit non seulement d'une propriété différentielle, mais surtout d'une appropriation des moyens de production par le capital, ce qui conduit la production à être subordonnée à ses objectifs. Pour Roemer, un régime de marchés concurrentiels suffit à produire exploitation et classes, et des préférences et des technologies différentielles remplacent l'échange de travail. L'exploitation n'est plus une question de rapport de domination de classe mais se réduit à une question de richesse individuelle : les agents sont exploiteurs ou exploités selon qu'ils se situent au-dessus ou en-dessous d'un certain seuil. Telle est la forme prise par l'inversion de l'ordre logique entre rapports de propriété et rapports de production : pour Roemer, une propriété différentielle des moyens de production conduit à l'exploitation capitaliste, tandis que pour Marx, les rapports de production capitalistes donnent naissance aux rapports de propriété capitalistes. Il considère que le rapport d'exploitation entre capital et travail est un postulat non prouvé par Marx. Il estime que le concept d'exploitation n'est pas d'un intérêt éthique fondamental et dans tous les cas, il semble nier que l'exploitation puisse renvoyer à une interaction entre individus. Il est donc possible d'établir une exploitation sans rapport de classe, et de décrire une structure de classes telle qu'elles sont déterminées par un processus d'optimisation individuelle.

Roemer a exercé une influence significative sur le Groupe de Septembre, notamment sur les travaux de Wright à propos des classes sociales (1985). Il intègre ainsi les outils de l'économie néoclassique, à

\footnotetext{
31 Notons à ce titre l'objection malicieuse de Daniel Bensaïd pour qui « nul [...] ne peut se retirer de la lutte des classes » (1995, p. 180).

32 Cette approche en termes de jeux coopératifs permet à Roemer d'insérer l'exploitation dans le champ de la justice distributive, vers lequel ses travaux se sont orientés à partir des années 1990. Voir notamment Roemer, 1996.

${ }^{33}$ Font exception linguistique les contributions de Marc Fleurbaey (1990) et d'André Hervier (1995).

${ }^{34}$ Une recension des critiques de la théorie de Roemer pourrait faire l'objet d'un autre article.
} 
commencer par l'individualisme méthodologique et la théorie des jeux, à l'approche épistémologique initiée par Cohen. Il associe sa reconstruction de la théorie de l'exploitation à la défense analytique du matérialisme historique par Cohen.

\subsection{Exploitation et matérialisme historique : l'influence de Cohen}

Roemer a inscrit sa théorie de l'exploitation dans le cadre de la conception marxienne de l'histoire, telle que présentée par Cohen (1978), i.e. une approche qui articule l'histoire par une relation entre forces productives et rapports de production. Pour Marx, la loi de la baisse tendancielle du taux de profit joue, dans le matérialisme historique, le rôle de mécanisme déclencheur de la transformation sociale. Pour Roemer, seul la diminution du taux d'exploitation peut être la cause de la baisse du taux de profit, et le matérialisme historique est traité comme un facteur exogène. Il élargit l'exploitation au-delà de la propriété des moyens de production, comme une simple forme historique de domination, ce qui lui permet d'envisager son existence dans des pays anciennement dits socialistes. Il se positionne dans le prolongement de Cohen (2.2.1), en associant la conception du matérialisme historique par ce dernier et la théorie des jeux (2.2.2).

\subsubsection{L'exploitation comme forme bistorique de domination, dans la continuité de Cohen}

L'objectif de Roemer est de construire une théorie générale de l'exploitation fondée sur le matérialisme historique, en adaptant les hypothèses aux sociétés de la fin du vingtième siècle. Le matérialisme historique serait la branche théorique du marxisme, celle qui lui apporte un contenu révolutionnaire ${ }^{35}$, indépendamment du reste du corpus. Roemer présente ses travaux comme un complément à ceux de Cohen, au sens où il considère que le matérialisme historique classique ne suffit pas à expliquer l'équilibre des rapports de classe. Il revendique explicitement cette continuité analytique et admet que le développement économique est associé au lien entre forces productives et rapports de production. Cohen présente une défense de la théorie de l'histoire de Marx dont la spécificité tient à son association avec «les normes de clarté et de rigueur de la philosophie analytique du vingtième siècle », (1978, ix). C'est ainsi qu'après une définition minutieuse des concepts qu'il juge centraux pour le matérialisme historique ${ }^{36}$, il articule la théorie avec un ensemble de thèses. D'une part, les forces productives se développementà travers l'histoire ; d'autre part, les forces productives détiennent une primauté explicative sur les rapports de production. Ainsi, lorsque le développement des forces productives est entravé, les rapports de production se transforment pour passer à une étape historique supérieure. L'histoire progresse ainsi par succession de concordances et de discordances entre rapports de production et de forces productives.

Roemer va chercher à inscrire sa théorie de l'exploitation dans le cadre de l'interprétation de Cohen. À un moindre niveau d'abstraction, sa position se situe à mi-chemin entre Cohen et Brenner (1982), c'est-à-dire qu'il ne choisit pas explicitement entre primauté des forces productives ou de la lutte de classes. Estimant que l'histoire progresse par élimination successive des formes d'exploitation devenant inutiles, il associe à chaque mode de production une forme d'exploitation. Ainsi il conceptualise la notion d'exploitation socialement nécessaire et il applique son modèle général à plusieurs types d'exploitation : féodale, capitaliste et socialiste. Il associe l'exploitation capitaliste à l'exploitation marxienne (sans contrainte apparente) et l'exploitation féodale 37 à l'exploitation néoclassique (avec contrainte apparente), et considère qu'une transition révolutionnaire a pour fonction d'éliminer la forme associée d'exploitation. L'existence de régimes autoritaires dans les États dits socialistes - en référence aux États où le capital a été exproprié - apparait pour Roemer comme une anomalie dans le programme de recherche marxiste, puisqu'il y voit un processus d'exploitation alors que la disparition du capitalisme était censée conduire à la suppression de cette exploitation. Un objectif est de fournir une théorie de l'exploitation en l'absence de propriété privée des moyens de production, c'est-à-dire dans les pays socialistes. Contre l'énoncé marxiste traditionnel selon lequel l'individualisme méthodologique et la théorie des jeux n'apportent rien au matérialisme historique, au sens où ils sont ahistoriques, Roemer répond que la théorie des jeux permetd'étudier les formes particulières de la lutte de classes spécifiée par le matérialisme historique, et donc que l'explication de cette lutte exige

\footnotetext{
35 «Le rôle révolutionnaire du marxisme [...] est un corollaire de sa théorie du matérialisme historique » (Roemer 1982a, p. 4).

${ }^{36}$ Cohen s'appuie quasi-exclusivement sur la Préface de la Contribution à la critique de l'économie politique, publié par Marx en 1859. Cohen en extrait notamment les « forces productives», les « rapports de production », « superstructure »... pour en faire une analyse détaillée.

${ }^{37}$ L'exploitation antique (maitre-esclave) est comprise comme un sous-ensemble de l'exploitation féodale
} 
des fondements microéconomiques, qui permettent de déterminer l'existence de situations d'équilibre : «je pense que la lutte de classe et donc l'individualisme méthodologique et l'analyse en termes de théorie des jeux en particulier sont centrales » (Roemer 1982b, p. 514). Roemer rejoint, dans une certaine mesure, les critiques de Jon Elster (1982) et il considère que l'explication fonctionnelle n'est d'aucune utilité pour une conception de l'histoire. D'une part, elle ne permet pas de comprendrele capitalisme tel qu'il s'est développé au vingtième siècle, au sens où elle ne propose pas d'explication pour les transitions historiques. D'autre part, elle n'apporte aucune information sur les développements futurs, c'est-à-dire sur le processus de convergence vers un nouvel équilibre. Reste qu'il présente son approche comme complémentaire à celle de Cohen.

\subsubsection{Théorie des jeux et mécanisme de transition}

Nous avons constaté que selon Roemer, l'exploitation existe dans une société donnée si un groupe peut améliorer sa situation matérielle en se retirant de cette société avec sa dotation (en force de travail, en biens aliénables ou en biens inaliénables selon la forme sociale). Il énonce plusieurs spécifications des règles de retrait, correspondant à chaque forme d'exploitation, c'est-à-dire à chaque jeu, à savoir l'exploitation féodale, capitaliste et socialiste, chacune d'entre elles étant associée à une inégalité de dotation et à des rapports de classe spécifiques, c'est-à-dire à un mode particulier de contrôle sur les biens productifs. Ainsi Roemer classifie les formes sociales en fonction du mode de contrôle sur les biens productifs, et donc du bien inégalement distribué.

\section{$\checkmark$ Une exploitation féodale a lieu en cas d'inégale propriété de la force de travail.}

L'exploitation apparait lorsque, s'il se retire, un serf dispose de sa dotation sans avoir à travailler pour le seigneur et la situation du seigneur se détériore. Il existe une coercition extra-économique évidente, une superstructure dont la fonction est d'extraire la force de travail. Une transformation révolutionnaire a donc pour tâche l'acquisition de libertés individuelles et l'établissement de la propriété privée.

$\checkmark$ Une exploitation capitaliste a lieu en cas d'inégale propriété des biens aliénables, c'est-à-dire les moyens de production.

Les prolétaires sont exploités, d'une part, s'ils ont intérêt à se retirer, d'autre part, si leur retrait détériore la situation des capitalistes. L'exploitation capitaliste résulte de la propriété privée des moyens de production et les rapports de propriété capitalistes obscurcissent la nature de l'appropriation du surplus du point de vue de l'ouvrier, puisqu'elle a lieu par le biais d'échanges marchands de force de travail et de marchandises. La distinction entre travail nécessaire et surtravail, et donc la coercition directe, ne sont pas observables; il reste une coercition extra-économique non évidente, l'État. Une transformation révolutionnaire a pour objectif la socialisation des moyens de production.

\section{$\checkmark$ Une exploitation socialiste a lieu en cas d'inégale dotation en biens inaliénables (qualifications ou statut).}

Les rapports de production se déroulent entre classe ouvrière et bureaucratie, ou entre classe ouvrière et experts selon que le socialisme est bureaucratique ou non. Un agent est exploité au sens socialiste si sa connaissance technologique (ou son pouvoir administratif) est limitée. Ce n'est pas une exploitation capitaliste car l'accès au capital social est le même pour tous. Sur ce point, Roemer se réfère à la bureaucratie des pays de l'Est. Une transformation révolutionnaire a donc pour objectif l'égalité réelle.

En évoluant de féodale à socialiste, une société traverse et élimine plusieurs formes d'exploitation, c'est-àdire plusieurs formes de rapports de propriété : le féodalisme correspond à l'exploitation féodale, capitaliste et socialiste. Il s'agit d'un jeu à trois classes : classe féodale, classe capitaliste et classe des producteurs directs. Toutes les formes d'exploitation existent sous le féodalisme. Sous le capitalisme, l'exploitation féodale devient interdite - les rapports féodaux ont été éliminés car inutiles au sens dynamique -, et le capitalisme correspond à l'exploitation capitaliste et socialiste : il s'agit d'un jeu à deux personnes, classe ouvrière et classe capitaliste. L'exploitation capitaliste devient socialement nécessaire puis elle se transforme en une entrave au développement des forces productives, devient inutile et doit être éliminée par une révolution socialiste. Le socialisme correspond à l'exploitation socialiste. Il s'agit d'un jeu à deux personnes, classe ouvrière et bureaucratie, ou classe ouvrière et experts. L'exploitation socialiste devient socialement nécessaire... Il s'agit d'un processus systématique et reproductif dans lequel chaque forme sociale élimine sa forme d'exploitation correspondante. 
Roemer met l'accent sur les périodes révolutionnaires, auxquelles Cohen attachait une moindre importance. Chaque révolution a pour tâche d'éliminer une forme spécifique d'exploitation, et les transitions historiques correspondent à différentes structures de jeux. Pour Marx, l'élimination du capitalisme est une condition nécessaire mais non suffisante au libre développement de tous et de chacun. La révolution communiste éliminera l'exploitation socialiste vers la satisfaction de la revendication «à chacun selon ses besoins 》 (Marx 1875, p. 32). L'exploitation est donc socialement nécessaire et le matérialisme historique énonce que l'histoire élimine les formes d'exploitation lorsqu'elles ne le sont plus. La tâche historique d'une époque est donc d'éliminer les entraves au développement des forces productives. Ce processus suit un ordre déterminé, jusqu'au communisme, ce qui renvoie à la logique déterministe attribuée à l'interprétation de Cohen ${ }^{38}$.

\section{Conclusion}

Contrairement à celle de Morishima, la reconstruction néoclassique de la théorie économique de Marx par Roemer vise à la préserver, au moins une partie, à savoir la théorie de l'exploitation, qui est ici présentée sous la forme d'un acte rationnel, sans avoir besoin de la théorie de la valeur travail. Pour Roemer il est possible de présenter le marxisme sous la forme des cinq énoncés suivants : les classes agissent comme des unités dans des situations historiques déterminées ; une position de classe se définit dans le rapport d'une personne aux moyens de production; la lutte de classes est le mécanisme du changement historique ; le changement historique suit une trajectoire déterminée ; le capitalisme est condamnable d'un point de vue éthique. Cela étant, il abandonne à la fois la théorie de la valeur travail, la loi de la baisse tendancielle du taux de profit, et donc le lien entre la théorie de l'exploitation et la théorie de la valeur travail. Ainsi l'exploitation est présentée comme un processus d'optimisation, ce qui fait perdre à la théorie à la fois son contenu matérialiste et une partie de son contenu subversif. Il a ainsi largement développéle programme de recherche marxiste analytique en complétant la lecture de Cohen sur la théorie de l'histoire par un travail sur la théorie économique. Pourtant, linterprétation qu'il propose de Marx porte à discussion, dans la mesure où il ne discute pas le postulat sur lequel il fonde son raisonnement, à savoir que les fondements méthodologiques de Marx sont erronés. Les résultats auxquels parvient Roemer militent sans ambiguité en faveur d'une remise en cause de l'aspect économique de la théorie de Marx, plus précisément d'une incompatibilité avec les outils de la théorie néoclassique. Nous pourrions ainsi décider d'abandonner soit la théorie économique de Marx soit l'interprétation qui en est faite de Roemer. Toutefois une telle conclusion nous semble prématurée, sachant que certaines approches paraissent moins tranchées dans l'opposition méthodologique entre marxisme et théorie néoclassique ${ }^{39}$. À ce titre, il nous semble productif de ne pas abandonner la spécificité de la méthode de Marx afin de poursuivre cet exercice visant à réfléchir la possibilité d'une association, ou du moins d'un dialogue, entre ces deux traditions.

\footnotetext{
${ }_{38}$ Par la suite Cohen prit ses distances avec cette approche. Voir Tarrit 2013.

${ }^{39}$ Voir notamment les contributions déjà mentionnées d'Erik O. Wright et de Nicholas Vrousalis. Voir également Wolff, Resnick 1988.
} 


\section{Références bibliographiques}

Anderson W.H. Locke et Thompson Frank W., « Neo-classical Marxism », Science and Society, vol. 52, n 2 , 1988, p. 215-228.

Bensaïd Daniel, Marx l'intempestif. Grandeurs et misères d'une aventure critique (XIXe-XXe siècles), Paris, Fayard, 1995, 416 p.

Brenner Robert, «The Agrarian Origins of European Capitalism », Past and Present, n 97, 1982, p. 16-113.

Burris Val, «New Directions in Class Analysis », Critical Sociology, vol. 15, n 1, 1988, p. 57-66.

Callinicos Alex, Making History: Agency, Structure and Change in Social Theory, Cambridge, Polity Press, $290 \mathrm{p}$.

Callinicos Alex, «Theory, History and Commitment: An Interview », Imprints: a journal of analytical socialism, vol. $6, n^{\circ} 1,2002$, p. 3-27.

Cohen Gerald A., KarlMarx's Theory of History: A Defense, Londres, Oxford University Press, 1978, 369 p.

Cohen, Gerald A., "The Structure of Proletariat Unfreedom », Pbilosopby and Public Affairs, vol. 12, $n^{\circ} 1$, 1983, p. 3-34.

Elster Jon, «Un marxisme anglais. A propos d'une nouvelle interprétation du matérialisme historique », Annales Economie Sociétés Civilisations, vol. 36, n 5, 1981, p. 745-757.

Elster Jon, «Marxism, Functionalism and Game Theory: The Case for Methodological Individualism », Theory and Society, vol. 11, n 3, 1982, p. 453-482.

Fleurbaey Marc, «Exploitation et inégalité : du côté du marxisme analytique (à propos de J. Roemer)», Actuel Marx, no 7, 1990, p. 116-130.

Hervier André, «Le concept d'exploitation à la croisée des chemins : Marx et Roemer », Économie et société, $\mathrm{n}^{\circ} 24,1995$, p. 98-118.

Hunt Emery K., «Analytical Marxism», in Roberts B. et Feiner S., Radical Economics, Londres, Kluwer Academic Publishers, 1992, p. 91-107.

Kieve Ronald A., «From Necessary Illusion to Rational Choice? A critique of neo-Marxist Rational Choice Theory », Theory and Society, vol. 15, n 4, 1986, p. 557-582.

Kliman Andrew J. et McGlone Ted, «The Transformation non-Problem and the non-Transformation Problem », Capital and Class, n 35,1988 , p. 56-83.

Lafargue Paul, « Karl Marx », Neue Zeit, vol. 9, n 1, 1891, p. 10-17.

Lassalle Ferdinand, Réponse publique au comité central à propos de la convocation d'un congrès général des ouvriers allemands à Leiprig, Zurich, 1863.

Lebowitz Michael A., «Is ‘Analytical Marxism’ a Marxism?», Science and Society, vol. 52, n², 1988, p. 191 214.

Marx Karl, Manuscrits de 1857-1858, Paris, Éditions Sociales, deux tomes, 1980 [1858].

Marx Karl, Le capital, Liwre troisième, Paris, Éditions Sociales, trois tomes, 1978 [1867-1894].

Marx Karl et Engels Friedrich, Critique des programmes de Gothaet d'Erfurt, Paris, Éditions Sociales, 1972 [1875], $158 \mathrm{p}$.

Morishima Michio, Marx's Economics: A Dual Theory of V alue and Growth, Cambridge, Cambridge University Press, 1973, 198 p.

Morishima, Michio, «Marx à la lumière de la théorie économique moderne », Economie appliquée, tome 28, n4, 1975 [1974], p. 693-721.

Okishio Nobuo, «Progrès technique et taux de profit», in Abraham-Frois G., L'économie classique. Nowvelles perspectives, Paris, Economica, 1984 [1961], p. 110-126.

Roemer John, Analytical Foundations of Marxian Economic Theory, New York, Cambridge University Press, 1981, 220 p. 
Roemer John, A General Theory of Exploitation and Class, Cambridge, Cambridge University Press, 1982a, 298 p.

Roemer John, «Methodological Individualism and Deductive Marxism », Theory and Society, vol. 11, $\mathrm{n}^{\circ} 4$, 1982b, p. 513-520.

Roemer John éd., Analytical Marxism, Cambridge, Cambridge University Press, 1986, 313 p.

Roemer John, Free to Lose: An Introduction to Marxist Economic Philosophy, Cambridge, Harvard University Press, 1988, 203 p.

Roemer John, Theories of Distributive Justice, Cambridge, Harvard University Press, 1996, 352 p.

Saad-Filho Alfredo, «Le soi-disant problème de la transformation », in Fine B. et Saad-Filho A., CA-PITAL! Introduction à l'économie politique de Marx, Paris, Raisons d'agir, 2012, p. 161-172.

Shibata Kei, «The Meaning of the Theory of Value in Theoretical Economics », Kyoto University Economic Review, vol. 8, n 2, 1933, p. 49-68.

Shibata Kei, "On the Law of Decline in the Rate of Profit», Kyoto University Economic Review, vol. 9, n¹, 1934, p. 61-75.

Sinclair Upton, The goose-step, a study of American education, à compte d'auteur, 1923, 498 p.

Smith Tony, «Roemer on Marx's theory of exploitation: Shortcomings of a Non-Dialectical Approach», Science and Society, vol. 53, n 3, 1989, p. 327-340.

Steedman Ian, Marx after Sraffa, Londres, New Left Books, 1977, 218 p.

Takamasu Akira et Matsui Akira éd., Analytical Marxism, Tokyo, Nakanishiya Press, 1999.

Tarrit Fabien, «Gerald A. Cohen (1941-2009) et le marxisme : apports et prise de distance », Revue de philosophie économique, vol. 14, n 2, 2013, p. 3-41.

Tarrit Fabien, Le marxisme analytique. Une introduction critique, Paris, Syllepses, 2014, 142 p.

Vrousalis Nicholas, «Exploitation, Vulnerability, and Social Domination», Pbilosopby and Public Affairs, vol. $41, \mathrm{n}^{\circ} 2,2013$, p. 131-157.

Wright Erik O., Classes, Londres, Verso, 1985., 344 p

Yoshihara Naokiet Veneziani Roberto, «The Theory of Exploitation as the Unequal Exchange of Labour », Economics \& Pbilosophy, vol. 34, n³, 2018, p. 381-409. 